\title{
Dynamics in a Lotka-Volterra Predator-Prey Model with Time-Varying Delays
}

\author{
Changjin $X u^{1}$ and Yusen $W u^{2}$ \\ ${ }^{1}$ Guizhou Key Laboratory of Economics System Simulation, School of Mathematics and Statistics, \\ Guizhou University of Finance and Economics, Guiyang 550004, China \\ ${ }^{2}$ School of Mathematics and Statistics, Henan University of Science and Technology, Luoyang 471023, China \\ Correspondence should be addressed to Changjin Xu; xcj403@126.com
}

Received 31 May 2013; Revised 18 August 2013; Accepted 18 August 2013

Academic Editor: Mark McKibben

Copyright (C) 2013 C. Xu and Y. Wu. This is an open access article distributed under the Creative Commons Attribution License, which permits unrestricted use, distribution, and reproduction in any medium, provided the original work is properly cited.

A Lotka-Volterra predator-prey model with time-varying delays is investigated. By using the differential inequality theory, some sufficient conditions which ensure the permanence and global asymptotic stability of the system are established. The paper ends with some interesting numerical simulations that illustrate our analytical predictions.

\section{Introduction}

In 1992, Berryman [1] pointed out that the dynamical relationship between predators and their prey has long been and will continue to be one of the dominant themes in both ecology and mathematical ecology due to its universal existence and importance. Dynamical behavior of predatorprey models has been studied by a lot of papers. It is well known that the investigation on predator-prey models not only focuses on the discussion of stability, periodic oscillatory, bifurcation, and chaos [2-26], but also involves many other dynamical behaviors such as permanence. In many applications, the nature of permanence is of great interest. Recently, Chen [27] investigated the permanence of a discrete $n$-species food-chain system with delays. Fan and Li [28] gave a theoretical study on permanence of a delayed ratiodependent predator-prey model with Holling type functional response. Chen [29] focused on the permanence and global attractivity of Lotka-Volterra competition system with feedback control. Zhao and Jiang [30] analyzed the permanence and extinction for nonautonomous Lotka-Volterra system. Teng et al. [31] addressed the permanence criteria for delayed discrete nonautonomous-species Kolmogorov systems. For more research on the permanence behavior of predator-prey models, one can see [32-40].
In 2010, Lv et al. [41] investigated the existence and global attractivity of periodic solution to the following LotkaVolterra predator-prey system:

$$
\begin{aligned}
& \frac{d x_{1}(t)}{d t} \\
& =x_{1}(t)\left[r_{1}(t)-a_{11}(t) x_{1}\left(t-\tau_{11}(t)\right)\right. \\
& \left.-a_{12}(t) x_{2}\left(t-\tau_{12}(t)\right)-a_{13}(t) x_{3}\left(t-\tau_{13}(t)\right)\right], \\
& \frac{d x_{2}(t)}{d t} \\
& =x_{2}(t)\left[-r_{2}(t)+a_{21}(t) x_{1}\left(t-\tau_{21}(t)\right)\right. \\
& \left.-a_{22}(t) x_{2}\left(t-\tau_{22}(t)\right)-a_{23}(t) x_{3}\left(t-\tau_{23}(t)\right)\right], \\
& \frac{d x_{3}(t)}{d t} \\
& =x_{3}(t)\left[-r_{3}(t)+a_{31}(t) x_{1}\left(t-\tau_{31}(t)\right)\right. \\
& \left.-a_{32}(t) x_{2}\left(t-\tau_{32}(t)\right)-a_{33}(t) x_{3}\left(t-\tau_{33}(t)\right)\right],
\end{aligned}
$$

where $x_{1}(t)$ denotes the density of prey species at time $t, x_{2}(t)$ and $x_{3}(t)$ stand for the density of predator species at time 
$t, r_{i}, a_{i j} \in C(\mathbb{R},[0, \infty))$ and $\tau_{i j} \in C(\mathbb{R}, \mathbb{R})$. Using Krasnoselskii's fixed point theorem and constructing Lyapunov function, Lv et al. obtained a set of easily verifiable sufficient conditions which guarantee the permanence and global attractivity of system (1).

For the viewpoint of biology, we shall consider (1) together with the initial conditions $x_{i}(0) \geq 0(i=1,2,3)$. The principle object of this paper is to explore the dynamics of system (1), applying the differential inequality theory to study the permanence of system (1). Using the method of Lyapunov function, we investigated the globally asymptotically stability of system (1).

The remainder of the paper is organized as follows: in Section 2, basic definitions and Lemmas are given, and some sufficient conditions for the permanence of the LotkaVolterra predator-prey model in consideration are established. A series of sufficient conditions for the global stability of the Lotka-Volterra predator-prey model in consideration are included in Section 3. In Section 4, we give an example which shows the feasibility of the main results. Conclusions are presented in Section 5.

\section{Permanence}

For convenience in the following discussing, we always use the notations:

$$
f^{l}=\inf _{t \in \mathbb{R}} f(t), \quad f^{u}=\sup _{t \in \mathbb{R}} f(t),
$$

where $f(t)$ is a continuous function. In order to obtain the main result of this paper, we shall first state the definition of permanence and several lemmas which will be useful in the proving the main result.

Definition 1 (see [41]). We say that system (1) is permanence if there are positive constants $M$ and $m$ such that for each positive solution $\left(x_{1}(t), x_{2}(t), x_{3}(t)\right)$ of system (1) satisfies

$$
m \leq \lim _{t \rightarrow+\infty} \inf x_{i}(t) \leq \lim _{t \rightarrow+\infty} \sup x_{i}(t) \leq M \quad(i=1,2,3) .
$$

Lemma 2 (see [42]). If $a>0, b>0$, and $\dot{x} \geq x(b-a x)$, when $t \geq 0$ and $x(0)>0$, we have

$$
\lim _{t \rightarrow+\infty} \inf x(t) \geq \frac{b}{a}
$$

If $a>0, b>0$, and $\dot{x} \leq x(b-a x)$, when $t \geq 0$ and $x(0)>0$, we have

$$
\lim _{t \rightarrow+\infty} \sup x(t) \leq \frac{b}{a}
$$

Now we state our permanence result for system (1).

Theorem 3. Let $M_{1}, M_{2}, M_{3}$, and $m_{1}$ be defined by (11), (18), (24), and (30), respectively. Suppose that the following conditions:

(H1) $a_{22}^{u} M_{1}>r_{2}^{l}, a_{31}^{u} M_{1}>r_{3}^{l}$,

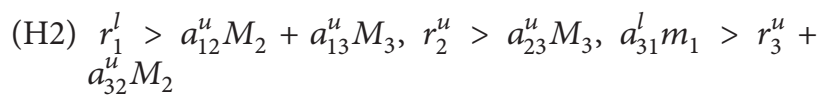

hold, and then system (1) is permanent; that is, there exist positive constants $m_{i}, M_{i}(i=1,2,3)$ which are independent of the solution of system (1), such that, for any positive solution $\left(x_{1}(t), x_{2}(t), x_{3}(t)\right)$ of system (1) with the initial condition $x_{i}(0) \geq 0(i=1,2,3)$, one has

$$
m_{i} \leq \lim _{t \rightarrow+\infty} \inf x_{i}(t) \leq \lim _{t \rightarrow+\infty} \sup x_{i}(t) \leq M_{i}
$$

Proof. It is easy to see that system (1) with the initial value condition $\left(x_{1}(0), x_{2}(0), x_{3}(0)\right)$ has positive solution $\left(x_{1}(t), x_{2}(t), x_{3}(t)\right)$ passing through $\left(x_{1}(0), x_{2}(0), x_{3}(0)\right)$. Let $\left(x_{1}(t), x_{2}(t), x_{3}(t)\right)$ be any positive solution of system (1) with the initial condition $\left(x_{1}(0), x_{2}(0), x_{3}(0)\right)$. It follows from the first equation of system (1) that

$$
\frac{d x_{1}(t)}{d t} \leq r_{1}(t) x_{1}(t) \leq r_{1}^{u} x_{1}(t)
$$

Integrating both sides of (7) from $t-\tau_{11}(t)$ to $t$, we get

$$
\ln \left[\frac{x_{1}(t)}{x_{1}\left(t-\tau_{11}(t)\right)}\right] \leq \int_{t-\tau_{11}(t)}^{t} r_{1}^{u} d s \leq r_{1}^{u} \tau_{11}^{u},
$$

which leads to

$$
x_{1}\left(t-\tau_{11}(t)\right) \geq x_{1}(t) \exp \left\{-r_{1}^{u} \tau_{11}^{u}\right\} .
$$

Substituting (9) into the first equation of system (1), it follows that

$$
\frac{d x_{1}(t)}{d t} \leq x_{1}(t)\left[r_{1}^{u}-a_{11}^{l} \exp \left\{-r_{1}^{u} \tau_{11}^{u}\right\} x_{1}(t)\right]
$$

It follows from (10) and Lemma 2 that

$$
\lim _{t \rightarrow+\infty} \sup x_{1}(t) \leq \frac{r_{1}^{u}}{a_{11}^{l}} \exp \left\{r_{1}^{u} \tau_{11}^{u}\right\}:=M_{1} .
$$

For any positive constant $\varepsilon>0$, it follows from (11) that there exists a $T_{1}>0$ such that, for all $t>T_{1}$,

$$
x_{1}(t) \leq M_{1}+\varepsilon
$$

For $t \geq T_{1}+\tau_{21}^{u}$, from (12) and the second equation of system (1), we have

$$
\begin{aligned}
\frac{d x_{2}(t)}{d t} & \leq x_{1}(t)\left[-r_{2}(t)+a_{21}(t) x_{1}\left(t-\tau_{21}(t)\right)\right] \\
& \leq x_{1}(t)\left[-r_{2}^{l}+a_{21}^{u}\left(M_{1}+\varepsilon\right)\right] .
\end{aligned}
$$

Integrating both sides of (13) from $t-\tau_{22}(t)$ to $t$, we get

$$
\begin{aligned}
\ln \left[\frac{x_{2}(t)}{x_{2}\left(t-\tau_{22}(t)\right)}\right] & \leq \int_{t-\tau_{22}(t)}^{t}\left[-r_{2}^{l}+a_{21}^{u}\left(M_{1}+\varepsilon\right)\right] d s \\
& \leq\left[-r_{2}^{l}+a_{21}^{u}\left(M_{1}+\varepsilon\right)\right] \tau_{22}^{u},
\end{aligned}
$$


which leads to

$$
x_{2}\left(t-\tau_{22}(t)\right) \geq x_{2}(t) \exp \left\{\left[r_{2}^{l}-a_{21}^{u}\left(M_{1}+\varepsilon\right)\right] \tau_{22}^{u}\right\} .
$$

Substituting (15) into the second equation of system (1), it follows that

$$
\begin{aligned}
\frac{d x_{2}(t)}{d t} \leq x_{2}(t)\{ & -r_{2}^{l}+a_{22}^{u}\left(M_{1}+\varepsilon\right) \\
& \left.-a_{22}^{l} \exp \left\{\left[r_{2}^{l}-a_{21}^{u}\left(M_{1}+\varepsilon\right)\right] \tau_{22}^{u}\right\} x_{2}(t)\right\} .
\end{aligned}
$$

Thus, as a direct corollary of Lemma 2, according to (16), one has

$$
\lim _{t \rightarrow+\infty} \sup x_{2}(t) \leq \frac{-r_{2}^{l}+a_{22}^{u}\left(M_{1}+\varepsilon\right)}{a_{22}^{l} \exp \left\{\left[r_{2}^{l}-a_{21}^{u}\left(M_{1}+\varepsilon\right)\right] \tau_{22}^{u}\right\}} .
$$

Setting $\varepsilon \rightarrow 0$, it follows that

$$
\lim _{t \rightarrow+\infty} \sup x_{2}(t) \leq \frac{-r_{2}^{l}+a_{22}^{u} M_{1}}{a_{22}^{l} \exp \left\{\left(r_{2}^{l}-a_{21}^{u} M_{1}\right) \tau_{22}^{u}\right\}}:=M_{2} .
$$

For $t \geq T_{1}+\tau_{31}^{u}$, from (12) and the third equation of system (1), we have

$$
\begin{aligned}
\frac{d x_{3}(t)}{d t} & \leq x_{3}(t)\left[-r_{3}(t)+a_{31}(t) x_{1}\left(t-\tau_{31}(t)\right)\right] \\
& \leq x_{3}(t)\left[-r_{3}^{l}+a_{31}^{u}\left(M_{1}+\varepsilon\right)\right] .
\end{aligned}
$$

Integrating both sides of (19) from $t-\tau_{33}(t)$ to $t$, we get

$$
\begin{aligned}
\ln \left[\frac{x_{3}(t)}{x_{3}\left(t-\tau_{33}(t)\right)}\right] & \leq \int_{t-\tau_{33}(t)}^{t}\left[-r_{3}^{l}+a_{31}^{u}\left(M_{1}+\varepsilon\right)\right] d s \\
& \leq\left[-r_{3}^{l}+a_{31}^{u}\left(M_{1}+\varepsilon\right)\right] \tau_{33}^{u},
\end{aligned}
$$

which leads to

$$
x_{3}\left(t-\tau_{33}(t)\right) \geq x_{3}(t) \exp \left\{\left[r_{3}^{l}-a_{31}^{u}\left(M_{1}+\varepsilon\right)\right] \tau_{33}^{u}\right\} .
$$

Substituting (21) into the third equation of system (1), it follows that

$$
\begin{aligned}
\frac{d x_{3}(t)}{d t} \leq x_{3}(t)\{ & \left\{-r_{3}^{l}+a_{31}^{u}\left(M_{1}+\varepsilon\right)\right. \\
& \left.-a_{33}^{l} \exp \left\{\left[r_{3}^{l}-a_{31}^{u}\left(M_{1}+\varepsilon\right)\right] \tau_{33}^{u}\right\} x_{3}(t)\right\} .
\end{aligned}
$$

Thus, as a direct corollary of Lemma 2, according to (22), one has

$$
\lim _{t \rightarrow+\infty} \sup x_{3}(t) \leq \frac{-r_{3}^{l}+a_{31}^{u}\left(M_{1}+\varepsilon\right)}{a_{33}^{l} \exp \left\{\left[r_{3}^{l}-a_{31}^{u}\left(M_{1}+\varepsilon\right)\right] \tau_{33}^{u}\right\}} .
$$

Setting $\varepsilon \rightarrow 0$, it follows that

$$
\lim _{t \rightarrow+\infty} \sup x_{3}(t) \leq \frac{-r_{3}^{l}+a_{31}^{u} M_{1}}{a_{33}^{l} \exp \left\{\left(r_{3}^{l}-a_{31}^{u} M_{1}\right) \tau_{33}^{u}\right\}}:=M_{3} .
$$

For $t \geq T_{1}+\max \left\{\tau_{21}^{u}, \tau_{31}^{u}, \tau_{11}^{u}, \tau_{12}^{u}, \tau_{13}^{u}\right\}$, it follows from the first equation of system (1) that

$$
\begin{aligned}
\frac{d x_{1}(t)}{d t} \geq x_{1}(t)[ & r_{1}^{l}-a_{11}^{u}\left(M_{1}+\varepsilon\right) \\
& \left.-a_{12}^{u}\left(M_{2}+\varepsilon\right)-a_{13}^{u}\left(M_{3}+\varepsilon\right)\right] .
\end{aligned}
$$

Integrating both sides of (25) from $t-\tau_{11}(t)$ to $t$, one has

$\ln \left[\frac{x_{1}(t)}{x_{1}\left(t-\tau_{11}(t)\right)}\right]$

$$
\begin{aligned}
& \geq \int_{t-\tau_{11}(t)}^{t}\left[r_{1}^{l}-a_{11}^{u}\left(M_{1}+\varepsilon\right)-a_{12}^{u}\left(M_{2}+\varepsilon\right)-a_{13}^{u}\left(M_{3}+\varepsilon\right)\right] d s \\
& \geq\left[r_{1}^{l}-a_{11}^{u}\left(M_{1}+\varepsilon\right)-a_{12}^{u}\left(M_{2}+\varepsilon\right)-a_{13}^{u}\left(M_{3}+\varepsilon\right)\right] \tau_{22}^{u},
\end{aligned}
$$

which leads to

$$
\begin{aligned}
& x_{1}\left(t-\tau_{11}(t)\right) \\
& \qquad x_{1}(t) \exp \left\{-\left[r_{1}^{l}-a_{11}^{u}\left(M_{1}+\varepsilon\right)\right.\right. \\
& \left.\left.\quad-a_{12}^{u}\left(M_{2}+\varepsilon\right)-a_{13}^{u}\left(M_{3}+\varepsilon\right)\right] \tau_{22}^{u}\right\} .
\end{aligned}
$$

Substituting (27) into the first equation of system (1), it follows that

$$
\begin{aligned}
& \frac{d x_{1}(t)}{d t} \\
& \geq x_{1}(t)\left\{r_{1}^{l}-a_{12}^{u}\left(M_{2}+\varepsilon\right)-a_{13}^{u}\left(M_{3}+\varepsilon\right)\right. \\
& -a_{11}^{u} \exp \left\{-\left[r_{1}^{l}-a_{11}^{u}\left(M_{1}+\varepsilon\right)\right.\right. \\
& \left.\quad-a_{12}^{u}\left(M_{2}+\varepsilon\right)-a_{13}^{u}\left(M_{3}+\varepsilon\right)\right] \\
& \left.\left.\times \tau_{22}^{u}\right\} x_{1}(t)\right\} .
\end{aligned}
$$

According to Lemma 2, it follows from (28) that

$$
\begin{aligned}
& \lim _{t \rightarrow+\infty} \inf x_{1}(t) \\
& \geq\left(r_{1}^{l}-a_{12}^{u}\left(M_{2}+\varepsilon\right)-a_{13}^{u}\left(M_{3}+\varepsilon\right)\right) \\
& \times\left(a _ { 1 1 } ^ { u } \operatorname { e x p } \left\{\left[-r_{1}^{l}-a_{11}^{u}\left(M_{1}+\varepsilon\right)\right.\right.\right. \\
& \left.\left.\left.\quad-a_{12}^{u}\left(M_{2}+\varepsilon\right)-a_{13}^{u}\left(M_{3}+\varepsilon\right)\right] \tau_{22}^{u}\right\}\right)^{-1} .
\end{aligned}
$$

Setting $\varepsilon \rightarrow 0$ in (29), we can get

$$
\begin{aligned}
& \lim _{t \rightarrow+\infty} \inf x_{1}(t) \\
& \geq \frac{r_{1}^{l}-a_{12}^{u} M_{2}-a_{13}^{u} M_{3}}{a_{11}^{u} \exp \left\{-\left(r_{1}^{l}-a_{11}^{u} M_{1}-a_{12}^{u} M_{2}-a_{13}^{u} M_{3}\right) \tau_{22}^{u}\right\}}:=m_{1} .
\end{aligned}
$$


For $t \geq T_{1}+\max \left\{\tau_{21}^{u}, \tau_{22}^{u}, \tau_{23}^{u}, \tau_{31}^{u}, \tau_{11}^{u}, \tau_{12}^{u}, \tau_{13}^{u}\right\}$, from the second equation of system (1), we have

$$
\begin{aligned}
& \frac{d x_{2}(t)}{d t} \\
& \geq x_{2}(t)\left[-r_{2}^{u}+a_{21}^{l}\left(m_{1}-\varepsilon\right)-a_{22}^{u}\left(M_{2}+\varepsilon\right)-a_{23}^{u}\left(M_{3}+\varepsilon\right)\right] .
\end{aligned}
$$

Integrating both sides of (31) from $t-\tau_{22}(t)$ to $t$ leads to

$$
\begin{aligned}
& \ln \left[\frac{x_{2}(t)}{x_{2}\left(t-\tau_{22}(t)\right)}\right] \\
& \geq \int_{t-\tau_{22}(t)}^{t}\left[-r_{2}^{u}+a_{21}^{l}\left(m_{1}-\varepsilon\right)\right. \\
& \left.\quad-a_{22}^{u}\left(M_{2}+\varepsilon\right)-a_{23}^{u}\left(M_{3}+\varepsilon\right)\right] d s \\
& \geq\left[-r_{2}^{u}+a_{21}^{l}\left(m_{1}-\varepsilon\right)-a_{22}^{u}\left(M_{2}+\varepsilon\right)-a_{23}^{u}\left(M_{3}+\varepsilon\right)\right] \tau_{22}^{u},
\end{aligned}
$$

which leads to

$$
\begin{gathered}
x_{2}\left(t-\tau_{22}(t)\right) \\
\leq x_{2}(t) \exp \left\{\left[r_{2}^{u}-a_{21}^{l}\left(m_{1}-\varepsilon\right)+a_{22}^{u}\left(M_{2}+\varepsilon\right)\right.\right. \\
\left.\left.+a_{23}^{u}\left(M_{3}+\varepsilon\right)\right] \tau_{22}^{u}\right\} .
\end{gathered}
$$

Substituting (33) into the second equation of system (1), it follows that

$$
\begin{aligned}
& \frac{d x_{2}(t)}{d t} \\
& \geq x_{2}(t)\left\{r_{2}^{u}-a_{22}^{u}\right. \\
& \quad \times \exp \left\{\left[r_{2}^{u}-a_{21}^{l}\left(m_{1}-\varepsilon\right)+a_{22}^{u}\left(M_{2}+\varepsilon\right)\right.\right. \\
& \left.\left.\left.\quad+a_{23}^{u}\left(M_{3}+\varepsilon\right)\right] \tau_{22}^{u}\right\} x_{2}(t)-a_{23}^{u}\left(M_{3}+\varepsilon\right)\right\} .
\end{aligned}
$$

By Lemma 2 and (34), we can get

$$
\begin{aligned}
& \lim _{t \rightarrow+\infty} \inf x_{2}(t) \\
& \geq\left(r_{2}^{u}-a_{23}^{u}\left(M_{3}+\varepsilon\right)\right) \\
& \quad \times\left(a _ { 2 2 } ^ { u } \operatorname { e x p } \left\{\left[r_{2}^{u}-a_{21}^{l}\left(m_{1}-\varepsilon\right)\right.\right.\right. \\
& \left.\left.\left.\quad+a_{22}^{u}\left(M_{2}+\varepsilon\right)+a_{23}^{u}\left(M_{3}+\varepsilon\right)\right] \tau_{22}^{u}\right\}\right)^{-1} .
\end{aligned}
$$

Setting $\varepsilon \rightarrow 0$ in the above inequality, it follows that

$$
\begin{aligned}
& \lim _{t \rightarrow+\infty} \inf x_{2}(t) \\
& \geq \frac{r_{2}^{u}-a_{23}^{u} M_{3}}{a_{22}^{u} \exp \left\{\left(r_{2}^{u}-a_{21}^{l} m_{1}+a_{22}^{u} M_{2}+a_{23}^{u} M_{3}\right) \tau_{22}^{u}\right\}}:=m_{2} .
\end{aligned}
$$

For $t \geq T_{1}+\max \left\{\tau_{31}^{u}, \tau_{32}^{u}, \tau_{33}^{u}, \tau_{21}^{u}, \tau_{22}^{u}, \tau_{23}^{u}, \tau_{31}^{u}, \tau_{11}^{u}, \tau_{12}^{u}, \tau_{13}^{u}\right\}$, it follows from the third equation of system (1) that

$$
\begin{aligned}
& \frac{d x_{3}(t)}{d t} \\
& =x_{3}(t)\left[-r_{3}(t)+a_{31}(t) x_{1}\left(t-\tau_{31}(t)\right)\right. \\
& \left.-a_{32}(t) x_{2}\left(t-\tau_{32}(t)\right)-a_{33}(t) x_{3}\left(t-\tau_{33}(t)\right)\right], \\
& \geq x_{3}(t)\left[-r_{3}^{u}+a_{31}^{l}\left(m_{1}-\varepsilon\right)\right. \\
& \left.-a_{32}^{u}\left(M_{2}+\varepsilon\right)-a_{33}^{u}\left(M_{3}+\varepsilon\right)\right]
\end{aligned}
$$

Integrating both sides of (37) from $t-\tau_{33}(t)$ to $t$, we get

$$
\begin{aligned}
& \ln \left[\frac{x_{3}(t)}{x_{3}\left(t-\tau_{33}(t)\right)}\right] \\
& \geq \int_{t-\tau_{33}(t)}^{t}\left[-r_{3}^{u}+a_{31}^{l}\left(m_{1}-\varepsilon\right)\right. \\
& \left.\quad-a_{32}^{u}\left(M_{2}+\varepsilon\right)-a_{33}^{u}\left(M_{3}+\varepsilon\right)\right] d s \\
& \geq\left[-r_{3}^{u}+a_{31}^{l}\left(m_{1}-\varepsilon\right)-a_{32}^{u}\left(M_{2}+\varepsilon\right)-a_{33}^{u}\left(M_{3}+\varepsilon\right)\right] \tau_{33}^{u} .
\end{aligned}
$$

Hence

$$
\begin{aligned}
& x_{3}\left(t-\tau_{33}(t)\right) \\
& \leq x_{3}(t) \exp \left\{\left[r_{3}^{u}-a_{31}^{l}\left(m_{1}-\varepsilon\right)\right.\right. \\
& \left.\left.\quad+a_{32}^{u}\left(M_{2}+\varepsilon\right)+a_{33}^{u}\left(M_{3}+\varepsilon\right)\right] \tau_{33}^{u}\right\} .
\end{aligned}
$$

Substituting (39) into the third equation of system (1), we derive

$$
\begin{aligned}
& \frac{d x_{3}(t)}{d t} \\
& \geq x_{3}(t)\left\{-r_{3}^{u}+a_{31}^{l}\left(m_{1}-\varepsilon\right)-a_{32}\left(M_{2}+\varepsilon\right)\right. \\
& \quad-a_{33} \exp \left\{\left[r_{3}^{u}-a_{31}^{l}\left(m_{1}-\varepsilon\right)+a_{32}^{u}\left(M_{2}+\varepsilon\right)\right.\right. \\
& \left.\left.\left.\quad+a_{33}^{u}\left(M_{3}+\varepsilon\right)\right] \tau_{33}^{u}\right\} x_{3}(t)\right\} .
\end{aligned}
$$

In view of Lemma 2 and (40), one has

$$
\begin{gathered}
\lim _{t \rightarrow+\infty} \inf x_{3}(t) \\
\geq\left(-r_{3}^{u}+a_{31}^{l}\left(m_{1}-\varepsilon\right)-a_{32}^{u}\left(M_{2}+\varepsilon\right)\right) \\
\quad \times\left(a _ { 3 3 } \operatorname { e x p } \left\{\left[r_{3}^{u}-a_{31}^{l}\left(m_{1}-\varepsilon\right)+a_{32}^{u}\left(M_{2}+\varepsilon\right)\right.\right.\right. \\
\left.\left.\left.+a_{33}^{u}\left(M_{3}+\varepsilon\right)\right] \tau_{33}^{u}\right\}\right)^{-1} .
\end{gathered}
$$


Setting $\varepsilon \rightarrow 0$ in (41) leads to

$$
\begin{aligned}
& \lim _{t \rightarrow+\infty} \inf x_{3}(t) \\
& \geq \frac{-r_{3}^{u}+a_{31}^{l} m_{1}-a_{32}^{u} M_{2}}{a_{33}^{u} \exp \left\{\left(r_{3}^{u}-a_{31}^{l} m_{1}+a_{32}^{u} M_{2}+a_{33}^{u} M_{3}\right) \tau_{33}^{u}\right\}}:=m_{3} .
\end{aligned}
$$

Equations (11), (18), (24), (30), (36), and (42) show that system (1) is permanent. The proof of Theorem 3 is complete.

\section{Global Asymptotically Stability of Positive Solutions}

In this section, we formulate the global asymptotically stability of positive solutions of system (1).

Definition 4. A bounded positive solution $\left(x_{1}^{*}(t), x_{2}^{*}(t)\right.$, $\left.x_{3}^{*}(t)\right)^{T}$ of system (1) is said to be globally asymptotically stable if, for any other positive bounded solution $\left(x_{1}(t)\right.$, $\left.x_{2}(t), x_{3}(t)\right)^{T}$ of system (1), the following equality holds:

$$
\lim _{t \rightarrow+\infty}\left[\sum_{i=1}^{3}\left|x_{i}(t)-x_{i}^{*}(t)\right|\right]=0 .
$$

Definition 5 (see [24]). Let $\widetilde{h}$ be a real number and $f$ be a nonnegative function defined on $[\tilde{h},+\infty)$ such that $f$ is integrable on $[\widetilde{h},+\infty)$ and is uniformly continuous on $[\widetilde{h},+\infty)$, then $\lim _{t \rightarrow+\infty} f(t)=0$.

Theorem 6. In addition to (H1)-(H2), assume further that

(H3) $\lim _{t \rightarrow \infty} \inf A_{i}(t)>0$,

where $A_{i}(i=1,2,3)$ are defined by (48), (49), and (50), respectively. Then system (1) has a unique positive solution $\left(x_{1}^{*}(t), x_{2}^{*}(t), x_{3}^{*}(t)\right)^{T}$ which is global attractivity.

Proof. According to the conclusion of Theorem 3, there exists $T>0$ and positive constants $m_{i}, M_{i}(i=1,2,3)$ such that

$$
m_{i}<x_{i}^{*}(t) \leq M_{i} \quad i=1,2,3, t>T .
$$

Define

$$
V(t)=\sum_{i=1}^{3}\left|\ln x_{i}^{*}(t)-\ln x_{i}(t)\right|
$$

Calculating the upper-right derivative of $V(t)$ along the solution of (1), it follows for $t \geq T$ that

$$
\begin{aligned}
D^{+} V(t)= & \sum_{i=1}^{3}\left(\frac{x_{i}^{* \prime}(t)}{x_{i}^{*}(t)}-\frac{x_{i}^{\prime}(t)}{x_{i}(t)}\right) \operatorname{sgn}\left(x_{i}^{*}(t)-x_{i}(t)\right) \\
= & \operatorname{sgn}\left(x_{1}^{*}(t)-x_{1}(t)\right) \\
& \times \sum_{i=1}^{3}-a_{1 i}(t)\left[x_{i}^{*}\left(t-\tau_{1 i}(t)\right)-x_{i}\left(t-\tau_{1 i}(t)\right)\right]
\end{aligned}
$$

$$
\begin{aligned}
& +\operatorname{sgn}\left(x_{2}^{*}(t)-x_{2}(t)\right) \\
& +\sum_{i=1}^{3}-a_{2 i}(t)\left[x_{i}^{*}\left(t-\tau_{2 i}(t)\right)-x_{i}\left(t-\tau_{2 i}(t)\right)\right] \\
& +\operatorname{sgn}\left(x_{3}^{*}(t)-x_{3}(t)\right) \\
& +\sum_{i=1}^{3}-a_{3 i}(t)\left[x_{i}^{*}\left(t-\tau_{3 i}(t)\right)-x_{i}\left(t-\tau_{3 i}(t)\right)\right] \\
& \leq-a_{11}(t)\left|x_{1}^{*}\left(t-\tau_{11}(t)\right)-x_{1}\left(t-\tau_{11}(t)\right)\right| \\
& +a_{12}(t)\left|x_{2}^{*}\left(t-\tau_{12}(t)\right)-x_{2}\left(t-\tau_{12}(t)\right)\right| \\
& +a_{13}(t)\left|x_{3}^{*}\left(t-\tau_{13}(t)\right)-x_{3}\left(t-\tau_{13}(t)\right)\right| \\
& +a_{21}(t)\left|x_{1}^{*}\left(t-\tau_{21}(t)\right)-x_{1}\left(t-\tau_{21}(t)\right)\right| \\
& -a_{22}(t)\left|x_{2}^{*}\left(t-\tau_{22}(t)\right)-x_{2}\left(t-\tau_{22}(t)\right)\right| \\
& +a_{23}(t)\left|x_{3}^{*}\left(t-\tau_{23}(t)\right)-x_{3}\left(t-\tau_{23}(t)\right)\right| \\
& +a_{31}(t)\left|x_{1}^{*}\left(t-\tau_{31}(t)\right)-x_{1}\left(t-\tau_{31}(t)\right)\right| \\
& +a_{32}(t)\left|x_{2}^{*}\left(t-\tau_{32}(t)\right)-x_{2}\left(t-\tau_{32}(t)\right)\right| \\
& -a_{33}(t)\left|x_{3}^{*}\left(t-\tau_{33}(t)\right)-x_{3}\left(t-\tau_{33}(t)\right)\right| .
\end{aligned}
$$

It follows that

$D^{+} V(t)$

$$
\begin{aligned}
\leq & -a_{11}(t)\left|x_{1}^{*}\left(t-\tau_{11}(t)\right)-x_{1}\left(t-\tau_{11}(t)\right)\right| \\
& +a_{12}(t)\left|x_{2}^{*}\left(t-\tau_{12}(t)\right)-x_{2}\left(t-\tau_{12}(t)\right)\right| \\
& +a_{13}(t)\left|x_{3}^{*}\left(t-\tau_{13}(t)\right)-x_{3}\left(t-\tau_{13}(t)\right)\right| \\
& +a_{21}(t)\left|x_{1}^{*}\left(t-\tau_{21}(t)\right)-x_{1}\left(t-\tau_{21}(t)\right)\right| \\
& -a_{22}(t)\left|x_{2}^{*}\left(t-\tau_{22}(t)\right)-x_{2}\left(t-\tau_{22}(t)\right)\right| \\
& +a_{23}(t)\left|x_{3}^{*}\left(t-\tau_{23}(t)\right)-x_{3}\left(t-\tau_{23}(t)\right)\right| \\
& +a_{31}(t)\left|x_{1}^{*}\left(t-\tau_{31}(t)\right)-x_{1}\left(t-\tau_{31}(t)\right)\right| \\
& +a_{32}(t)\left|x_{2}^{*}\left(t-\tau_{32}(t)\right)-x_{2}\left(t-\tau_{32}(t)\right)\right| \\
& -a_{33}(t)\left|x_{3}^{*}\left(t-\tau_{33}(t)\right)-x_{3}\left(t-\tau_{33}(t)\right)\right| \\
\leq & -a_{11}(t)\left\{\operatorname { e x p } \left\{\left[r_{1}^{l}-a_{11}^{u}\left(M_{1}+\varepsilon\right)-a_{12}^{u}\left(M_{2}+\varepsilon\right)\right.\right.\right. \\
& \left.\left.\left.\quad-a_{13}^{u}\left(M_{3}+\varepsilon\right)\right] \tau_{22}^{u}\right\}+\exp \left\{-r^{u} \tau_{11}^{u}\right\}\right\} \\
& \times\left|x_{2}^{*}(t)-x_{2}(t)\right| \\
& +2 a_{12}(t) \exp \left\{\left[-x_{2}^{u}+a_{21}^{l}\left(m_{1}-\varepsilon\right)-a_{22}^{u}\left(M_{2}+\varepsilon\right)\right] \tau_{22}^{u}\right\} \\
& \quad x_{1}(t) \mid
\end{aligned}
$$




$$
\begin{aligned}
& +2 a_{13}(t) \exp \left\{\left[-r_{3}^{u}+a_{31}^{l}\left(m_{1}-\varepsilon\right)-a_{32}^{u}\left(M_{2}+\varepsilon\right)\right.\right. \\
& \left.\left.-a_{33}^{u}\left(M_{3}+\varepsilon\right)\right] \tau_{33}^{u}\right\} \\
& \times\left|x_{3}^{*}(t)-x_{3}(t)\right| \\
& +2 a_{21}(t) \exp \left\{-\left[r_{1}^{l}-a_{11}^{u}\left(M_{1}+\varepsilon\right)\right.\right. \\
& \left.\left.-a_{12}^{u}\left(M_{2}+\varepsilon\right)-a_{13}^{u}\left(M_{3}+\varepsilon\right)\right] \tau_{22}^{u}\right\} \\
& \times\left|x_{1}^{*}(t)-x_{1}(t)\right| \\
& -a_{22}(t)\left\{\operatorname { e x p } \left\{\left[-r_{2}^{u}+a_{21}^{l}\left(m_{1}-\varepsilon\right)-a_{22}^{u}\left(M_{2}+\varepsilon\right)\right.\right.\right. \\
& \left.\left.-a_{23}^{u}\left(M_{3}+\varepsilon\right)\right] \tau_{22}^{u}\right\} \\
& \left.+\exp \left\{\left[r_{2}^{l}-a_{21}^{u}\left(M_{1}+\varepsilon\right)\right] \tau_{22}^{u}\right\}\right\} \\
& \times\left|x_{2}^{*}(t)-x_{2}(t)\right| \\
& +2 a_{23}(t) \exp \left\{\left[r_{3}^{u}-a_{31}^{l}\left(m_{1}-\varepsilon\right)\right.\right. \\
& \left.\left.+a_{32}^{u}\left(M_{2}+\varepsilon\right)+a_{33}^{u}\left(M_{3}+\varepsilon\right)\right] \tau_{33}^{u}\right\} \\
& \times\left|x_{3}^{*}(t)-x_{3}(t)\right| \\
& +2 a_{31}(t) \exp \left\{-\left[r_{1}^{l}-a_{11}^{u}\left(M_{1}+\varepsilon\right)\right.\right. \\
& \left.\left.-a_{12}^{u}\left(M_{2}+\varepsilon\right)-a_{13}^{u}\left(M_{3}+\varepsilon\right)\right] \tau_{22}^{u}\right\} \\
& \times\left|x_{1}^{*}(t)-x_{1}(t)\right| \\
& +2 a_{32}(t) \exp \left\{\left[-r_{2}^{u}+a_{21}^{l}\left(m_{1}-\varepsilon\right)\right.\right. \\
& \left.\left.-a_{22}^{u}\left(M_{2}+\varepsilon\right)-a_{23}^{u}\left(M_{3}+\varepsilon\right)\right] \tau_{22}^{u}\right\} \\
& \times\left|x_{2}^{*}(t)-x_{2}(t)\right| \\
& -a_{33}(t)\left\{\operatorname { e x p } \left\{\left[-r_{3}^{u}+a_{31}^{l}\left(m_{1}-\varepsilon\right)\right.\right.\right. \\
& \left.\left.-a_{32}^{u}\left(M_{2}+\varepsilon\right)-a_{33}^{u}\left(M_{3}+\varepsilon\right)\right] \tau_{33}^{u}\right\} \\
& \left.+\exp \left\{\left[r_{3}^{l}-a_{31}^{u}\left(M_{1}+\varepsilon\right)\right] \tau_{33}^{u}\right\}\right\} \\
& \times\left|x_{3}^{*}(t)-x_{3}(t)\right| \\
& \leq\left[-A_{1}(t)\left|x_{1}^{*}(t)-x_{1}(t)\right|+A_{2}(t)\left|x_{2}^{*}(t)-x_{2}(t)\right|\right. \\
& \left.+A_{3}\left|x_{3}^{*}(t)-x_{3}(t)\right|\right] \text {, }
\end{aligned}
$$

where $\varepsilon$ is defined by Theorem 3 and

$$
\begin{aligned}
& A_{1}(t) \\
& =a_{11}(t)\left\{\operatorname { e x p } \left\{\left[r_{1}^{l}-a_{11}^{u}\left(M_{1}+\varepsilon\right)-a_{12}^{u}\left(M_{2}+\varepsilon\right)\right.\right.\right. \\
& \left.\left.\left.-a_{13}^{u}\left(M_{3}+\varepsilon\right)\right] \tau_{22}^{u}\right\}+\exp \left\{-r^{u} \tau_{11}^{u}\right\}\right\} \\
& -2 a_{21}(t) \exp \left\{-\left[r_{1}^{l}-a_{11}^{u}\left(M_{1}+\varepsilon\right)-a_{12}^{u}\left(M_{2}+\varepsilon\right)\right.\right. \\
& \left.\left.-a_{13}^{u}\left(M_{3}+\varepsilon\right)\right] \tau_{22}^{u}\right\} \\
& -2 a_{31}(t) \exp \left\{\tau_{22}^{u}-\left[r_{1}^{l}-a_{11}^{u}\left(M_{1}+\varepsilon\right)-a_{12}^{u}\left(M_{2}+\varepsilon\right)\right.\right. \\
& \left.\left.-a_{13}^{u}\left(M_{3}+\varepsilon\right)\right] \tau_{22}^{u}\right\}
\end{aligned}
$$

$$
\begin{aligned}
& A_{2}(t) \\
& =a_{22}(t)\left\{\operatorname { e x p } \left\{\left[-r_{2}^{u}+a_{21}^{l}\left(m_{1}-\varepsilon\right)-a_{22}^{u}\left(M_{2}+\varepsilon\right)\right.\right.\right. \\
& \left.\left.-a_{23}^{u}\left(M_{3}+\varepsilon\right)\right] \tau_{22}^{u}\right\} \\
& \left.+\exp \left\{\left[r_{2}^{l}-a_{21}^{u}\left(M_{1}+\varepsilon\right)\right] \tau_{22}^{u}\right\}\right\} \\
& -2 a_{12}(t) \exp \left\{\left[-r_{2}^{u}+a_{21}^{l}\left(m_{1}-\varepsilon\right)-a_{22}^{u}\left(M_{2}+\varepsilon\right)\right.\right. \\
& \left.\left.-a_{23}^{u}\left(M_{3}+\varepsilon\right)\right] \tau_{22}^{u}\right\} \\
& -2 a_{32}(t) \exp \left\{\left[-r_{2}^{u}+a_{21}^{l}\left(m_{1}-\varepsilon\right)-a_{22}^{u}\left(M_{2}+\varepsilon\right)\right.\right. \\
& \left.\left.-a_{23}^{u}\left(M_{3}+\varepsilon\right)\right] \tau_{22}^{u}\right\},
\end{aligned}
$$

$A_{3}(t)$

$$
\begin{aligned}
&=a_{33}(t)\{\exp \{[-r_{3}^{u}+a_{31}^{l}\left(m_{1}-\varepsilon\right) \\
&+\exp \left\{\left[r_{3}^{l}-a_{31}^{u}\left(M_{1}+\varepsilon\right)\right] \tau_{33}^{u}\right\} \\
&\left.\left.-a_{32}^{u}\left(M_{2}+\varepsilon\right)-a_{33}^{u}\left(M_{3}+\varepsilon\right)\right] \tau_{33}^{u}\right\} \\
&+\exp \left\{\left[r_{3}^{l}-a_{31}^{u}\left(M_{1}+\varepsilon\right)\right] \tau_{33}^{u}\right\} \\
&-2 a_{13}(t) \exp \left\{\left[-r_{3}^{u}+a_{31}^{l}\left(m_{1}-\varepsilon\right)-a_{32}^{u}\left(M_{2}+\varepsilon\right)\right.\right. \\
&\left.\left.\quad-a_{33}^{u}\left(M_{3}+\varepsilon\right)\right] \tau_{33}^{u}\right\} \\
&-2 a_{23}(t) \exp \left\{\left[r_{3}^{u}-a_{31}^{l}\left(m_{1}-\varepsilon\right)+a_{32}^{u}\left(M_{2}+\varepsilon\right)\right.\right. \\
&\left.\left.\quad+a_{33}^{u}\left(M_{3}+\varepsilon\right)\right] \tau_{33}^{u}\right\} .
\end{aligned}
$$

By hypothesis (H3), there exist constants $\alpha_{i}(i=1,2,3)$ and $T^{*}>T$ such that

$$
A_{i}(t) \geq \alpha_{i}>0, \quad(i=1,2,3) \text { for } t \geq T^{*} .
$$

Integrating both sides of $(51)$ on interval $\left[T^{*}, t\right]$ yields

$$
V(t)+\sum_{i=1}^{3} \int_{T^{*}}^{t} A_{i}(t)\left|x_{i}^{*}(t)-x_{i}(t)\right| d s \leq V\left(T^{*}\right) .
$$

It follows from (51) and (52) that

$\sum_{i=1}^{3} \int_{T^{*}}^{t} A_{i}(t)\left|x_{i}^{*}(t)-x_{i}(t)\right| d s \leq V\left(T^{*}\right)<\infty, \quad$ for $t \geq T^{*}$.

Since $x_{i}^{*}(t)(i=1,2,3)$ are bounded for $t \geq T^{*}$, so $\mid x_{i}^{*}(t)-$ $x_{i}(t) \mid(i, j=1,2,3)$ are uniformly continuous on $\left[T^{*}, \infty\right)$. By Barbalat's Lemma [24], we have

$$
\lim _{t \rightarrow \infty}\left|x_{i}^{*}(t)-x_{i}(t)\right|=0, \quad(i=1,2,3) .
$$

By Theorems 7.4 and 8.2 in [43], we know that the positive solution $\left(x_{1}^{*}(t), x_{2}^{*}(t), x_{3}^{*}(t)\right)^{T}$ of (1) is uniformly asymptotically stable. The proof of Theorem 6 is complete. 


\section{Numerical Example}

To illustrate the theoretical results, we present some numerical simulations. Let us consider the following discrete system:

$$
\begin{aligned}
\frac{d x_{1}(t)}{d t} & \\
=x_{1}(t)[ & 5-\frac{\cos \pi t}{2} \\
& -\left(4+\frac{\cos \pi t}{5}\right) x_{1}\left(t-\left(1-\frac{\sin \pi t}{4}\right)\right) \\
& -\left(\frac{1+\sin \pi t}{4}\right) x_{2}\left(t-\left(\frac{0.5-\sin \pi t}{4}\right)\right) \\
& \left.-\left(\frac{1+\cos \pi t}{3}\right) x_{3}\left(t-\left(\frac{0.9-\cos \pi t}{4}\right)\right)\right],
\end{aligned}
$$

$\frac{d x_{2}(t)}{d t}$

$$
\begin{aligned}
=x_{2}(t)[ & -\left(\frac{48-\cos \pi t}{12}\right) \\
& +\left(2-\frac{\cos \pi t}{4}\right) x_{1}\left(t-\left(\frac{0.7-\cos \pi t}{5}\right)\right) \\
& -\left(4-\frac{\cos \pi t}{12}\right) x_{2}\left(t-\left(\frac{1+\sin \pi t}{4}\right)\right) \\
& \left.-\left(1+\frac{\sin \pi t}{4}\right) x_{3}\left(t-\left(\frac{0.2-\sin \pi t}{12}\right)\right)\right],
\end{aligned}
$$

$\frac{d x_{3}(t)}{d t}$

$$
\begin{aligned}
=x_{3}(t)[ & -\left(\frac{1-\cos \pi t}{4}\right) \\
& +\left(8+\frac{\sin \pi t}{4}\right) x_{1}\left(t-\left(\frac{0.8-\sin \pi t}{5}\right)\right) \\
& -\left(\frac{0.6-\sin \pi t}{8}\right) x_{2}\left(t-\left(\frac{0.6-\cos \pi t}{12}\right)\right) \\
& \left.-\left(20+\frac{\sin \pi t}{4}\right) x_{3}\left(t-\left(0.5+\frac{\sin \pi t}{2}\right)\right)\right] .
\end{aligned}
$$

Here

$$
\begin{array}{rlrl}
r_{1}(t) & =5-\frac{\cos \pi t}{2}, & r_{2}(t)=\frac{48-\cos \pi t}{12}, \\
r_{3}(t)=\frac{2-\cos \pi t}{4}, & a_{11}(t)=4+\frac{\cos \pi t}{5}, \\
a_{12}(t)=\frac{1+\sin \pi t}{4}, & a_{13}(t)=\frac{1+\cos \pi t}{3}, \\
a_{21}(t)=2-\frac{\cos \pi t}{4}, & a_{22}(t)=4-\frac{\cos \pi t}{12}, \\
a_{23}(t)=1+\frac{\sin \pi t}{4}, & a_{31}(t)=8+\frac{\sin \pi t}{4}, \\
a_{32}(t)=\frac{0.6-\sin \pi t}{8}, & a_{33}(t)=20+\frac{\sin \pi t}{4}, \\
\tau_{11}(t)=1-\frac{\sin \pi t}{4}, & \tau_{12}(t)=\frac{0.5-\sin \pi t}{4}, \\
\tau_{13}(t)=\frac{0.9-\cos \pi t}{4}, & \tau_{21}(t)=\frac{0.7-\cos \pi t}{5},
\end{array}
$$

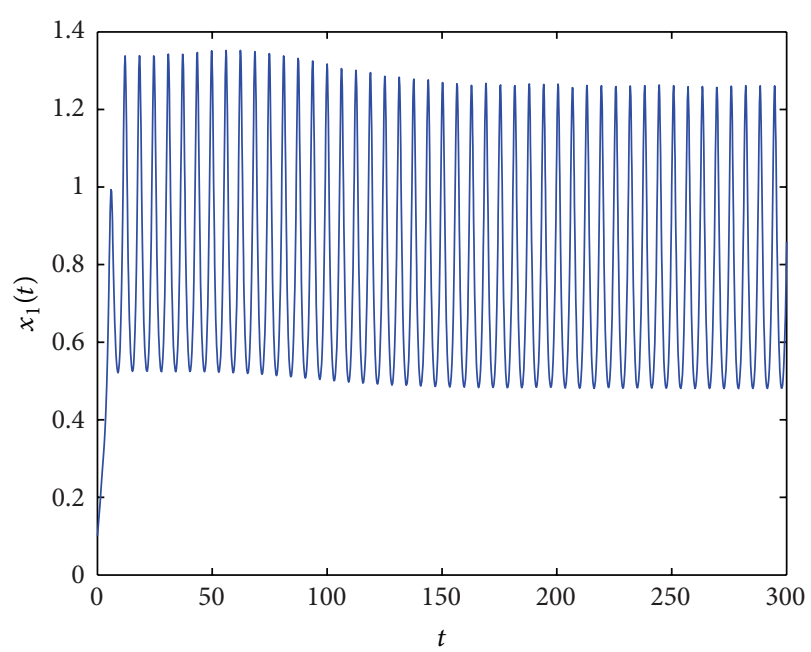

FIGURE 1: The dynamical behavior of the first component of the solution $\left(x_{1}(t), x_{2}(t), x_{3}(t)\right)$.

$$
\begin{gathered}
\tau_{22}(t)=\frac{1+\sin \pi t}{4}, \quad \tau_{23}(t)=\frac{0.2-\sin \pi t}{12}, \\
\tau_{31}(t)=\frac{0.8-\sin \pi t}{5}, \quad \tau_{32}(t)=\frac{0.6-\cos \pi t}{12}, \\
\tau_{33}(t)=0.5+\frac{\sin \pi t}{2} .
\end{gathered}
$$

All the coefficients $r_{i}(t)(i=1,2,3), a_{i j}(t)(i, j=1,2,3)$, $\tau_{i j}(t)(i, j=1,2,3)$ are functions with respect to $t$, and it is easy to see that

$$
\begin{aligned}
a_{22}^{u}=\frac{49}{12}, & a_{31}^{u}=\frac{33}{4}, & r_{2}^{l}=\frac{47}{12}, \\
r_{3}^{l}=\frac{1}{4}, & a_{12}^{u}=\frac{1}{2}, & a_{13}^{u}=\frac{2}{3}, \\
r_{2}^{u}=\frac{49}{12}, & a_{23}^{u}=\frac{5}{4}, & a_{31}^{l}=\frac{31}{4}, \\
r_{3}^{u}=\frac{3}{4}, & a_{32}^{u}=0.2, & r_{1}^{u}=5.5, \\
a_{11}^{l}=3.8, & \tau_{11}^{u}=1.25, & a_{22}^{l}=\frac{47}{12}, \\
a_{21}^{u}=2.25, & \tau_{22}^{u}=0.5, & a_{33}^{l}=19.75 .
\end{aligned}
$$

Then $M_{1}=1.2451, M_{2}=0.7395, M_{3}=2.1093, m_{1}=$ 0.6422 . Thus it is easy to see that all the conditions of Theorem 6 are satisfied. Thus system (55) is permanent which is shown in Figures 1, 2, and 3.

\section{Conclusions}

In this paper, we have investigated the dynamical behavior of a Lotka-Volterra predator-prey model with time-varying 


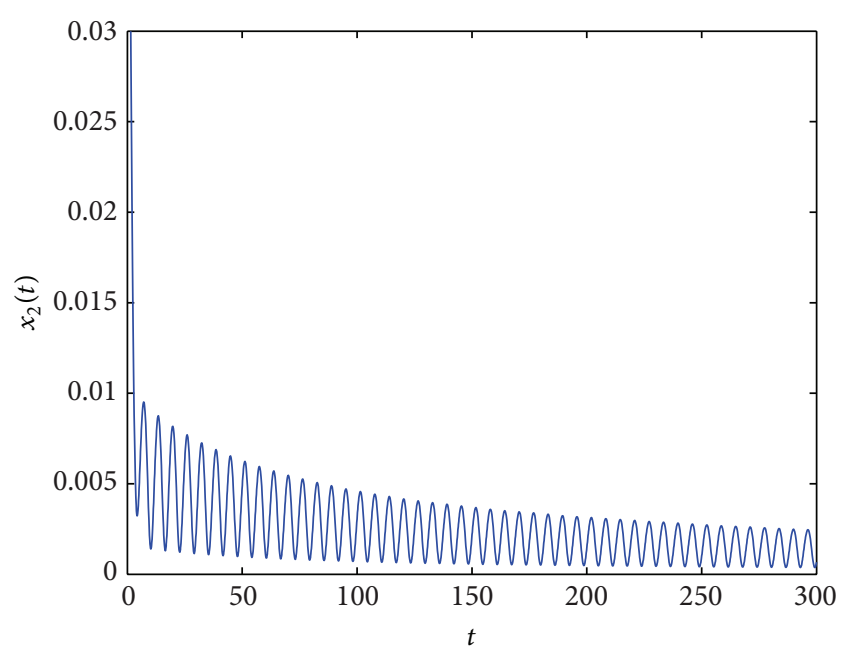

FIGURE 2: The dynamical behavior of the second component of the solution $\left(x_{1}(t), x_{2}(t), x_{3}(t)\right)$.

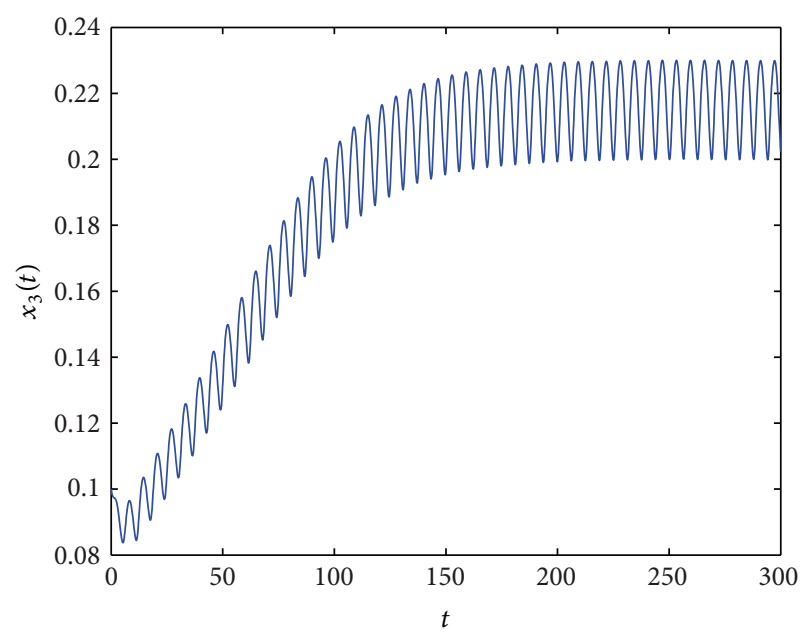

FIGURE 3: The dynamical behavior of the third component of the solution $\left(x_{1}(t), x_{2}(t), x_{3}(t)\right)$.

delays. Sufficient conditions which ensure the permanence of the system are derived. Moreover, we also deal with the global stability of the system. It is shown that delay has influence on the permanence and the global stability of system. Thus delay is an important factor to decide the permanence and global stability of the system. Numerical simulations show the feasibility of our main results.

\section{Acknowledgments}

This work is supported by National Natural Science Foundation of China (no. 11261010 and no. 11101126), Soft Science and Technology Program of Guizhou Province (no. 2011LKC2030), Natural Science and Technology Foundation of Guizhou Province (J[2012]2100), Governor Foundation of Guizhou Province ([2012]53), and Doctoral Foundation of Guizhou University of Finance and Economics (2010).

\section{References}

[1] A. A. Berryman, "The origins and evolution of predator-prey theory," Ecology, vol. 73, no. 5, pp. 1530-1535, 1992.

[2] B. Dai and J. Zou, "Periodic solutions of a discrete-time diffusive system governed by backward difference equations," Advances in Difference Equations, vol. 2005, no. 3, pp. 263-274, 2005.

[3] M. Gyllenberg, P. Yan, and Y. Wang, "Limit cycles for competitor-competitor-mutualist Lotka-Volterra systems," Physica D, vol. 221, no. 2, pp. 135-145, 2006.

[4] M. Fan and K. Wang, "Periodic solutions of a discrete time nonautonomous ratio-dependent predator-prey system," Mathematical and Computer Modelling, vol. 35, no. 9-10, pp. 951-961, 2002.

[5] M. Fazly and M. Hesaaraki, "Periodic solutions for a discrete time predator-prey system with monotone functional responses," Comptes Rendus Mathématique, vol. 345, no. 4, pp. 199-202, 2007.

[6] R. E. Gaines and J. L. Mawhin, Coincidence Degree, and Nonlinear Differential Equations, vol. 568 of Lecture Notes in Mathematics, Springer, Berlin, Germany, 1977.

[7] Y. Li, "Positive periodic solutions of a discrete mutualism model with time delays," International Journal of Mathematics and Mathematical Sciences, vol. 2005, no. 4, pp. 499-506, 2005.

[8] M. Sen, M. Banerjee, and A. Morozov, "Bifurcation analysis of a ratio-dependent prey-predator model with the Allee effect," Ecological Complexity, vol. 11, pp. 12-27, 2012.

[9] M. Haque and E. Venturino, "An ecoepidemiological model with disease in predator: the ratio-dependent case," Mathematical Methods in the Applied Sciences, vol. 30, no. 14, pp. 1791-1809, 2007.

[10] L.-L. Wang, W.-T. Li, and P.-H. Zhao, "Existence and global stability of positive periodic solutions of a discrete predatorprey system with delays," Advances in Difference Equations, vol. 2004, no. 4, pp. 321-336, 2004.

[11] J. Wiener, "Differential equations with piecewise constant delays," in Trends in Theory and Practice of Nonlinear Differential Equations, vol. 90 of Lecture Notes in Pure and Applied Mathematics, pp. 547-552, Dekker, New York, NY, USA, 1984.

[12] R. Xu, L. Chen, and F. Hao, "Periodic solutions of a discrete time Lotka-Volterra type food-chain model with delays," Applied Mathematics and Computation, vol. 171, no. 1, pp. 91-103, 2005.

[13] J. Zhang and H. Fang, "Multiple periodic solutions for a discrete time model of plankton allelopathy," Advances in Difference Equations, vol. 2006, Article ID 090479, 2006.

[14] X. Xiong and Z. Zhang, "Periodic solutions of a discrete twospecies competitive model with stage structure," Mathematical and Computer Modelling, vol. 48, no. 3-4, pp. 333-343, 2008.

[15] R. Y. Zhang, Z. C. Wang, Y. Chen, and J. Wu, "Periodic solutions of a single species discrete population model with periodic harvest/stock," Computers \& Mathematics with Applications, vol. 39, no. 1-2, pp. 77-90, 2000.

[16] W. Zhang, D. Zhu, and P. Bi, "Multiple positive periodic solutions of a delayed discrete predator-prey system with type IV functional responses," Applied Mathematics Letters, vol. 20, no. 10, pp. 1031-1038, 2007.

[17] Z. Zhang and J. Luo, "Multiple periodic solutions of a delayed predator-prey system with stage structure for the predator," Nonlinear Analysis: Real World Applications, vol. 11, no. 5, pp. 4109-4120, 2010. 
[18] Y. Li, K. Zhao, and Y. Ye, "Multiple positive periodic solutions of $n$ species delay competition systems with harvesting terms," Nonlinear Analysis: Real World Applications, vol. 12, no. 2, pp. 1013-1022, 2011.

[19] Y. G. Sun and S. H. Saker, "Positive periodic solutions of discrete three-level food-chain model of Holling type II," Applied Mathematics and Computation, vol. 180, no. 1, pp. 353-365, 2006.

[20] X. Ding and C. Lu, "Existence of positive periodic solution for ratio-dependent $N$-species difference system," Applied Mathematical Modelling, vol. 33, no. 6, pp. 2748-2756, 2009.

[21] K. Chakraborty, M. Chakraborty, and T. K. Kar, "Bifurcation and control of a bioeconomic model of a prey-predator system with a time delay," Nonlinear Analysis: Hybrid Systems, vol. 5, no. 4, pp. 613-625, 2011.

[22] Z.C. Li, Q.L. Zhao, and D. Ling, "Chaos in a discrete delay population model," Discrete Dynamics in Nature and Society, vol. 2012, Article ID 482459, 14 pages, 2012.

[23] H. Xiang, K.-M. Yan, and B.-Y. Wang, "Existence and global stability of periodic solution for delayed discrete high-order hopfield-type neural networks," Discrete Dynamics in Nature and Society, vol. 2005, no. 3, pp. 281-297, 2005.

[24] K. Gopalsamy, Stability and Oscillations in Delay Differential Equations of Population Dynamics, vol. 74 of Mathematics and its Applications, Kluwer Academic, Dordrecht, Netherlands, 1992.

[25] Y. Kuang, Delay Differential Equations with Applications in Population Dynamics, vol. 191 of Mathematics in Science and Engineering, Academic Press, New York, NY, USA, 1993.

[26] L. Fan, Z. Shi, and S. Tang, "Critical values of stability and Hopf bifurcations for a delayed population model with delaydependent parameters," Nonlinear Analysis: Real World Applications, vol. 11, no. 1, pp. 341-355, 2010.

[27] F. Chen, "Permanence of a discrete $n$-species food-chain system with time delays," Applied Mathematics and Computation, vol. 185, no. 1, pp. 719-726, 2007.

[28] Y.-H. Fan and W.-T. Li, "Permanence for a delayed discrete ratio-dependent predator-prey system with Holling type functional response," Journal of Mathematical Analysis and Applications, vol. 299, no. 2, pp. 357-374, 2004.

[29] F. Chen, "The permanence and global attractivity of LotkaVolterra competition system with feedback controls," Nonlinear Analysis: Real World Applications, vol. 7, no. 1, pp. 133-143, 2006.

[30] J. Zhao and J. Jiang, "Average conditions for permanence and extinction in nonautonomous Lotka-Volterra system," Journal of Mathematical Analysis and Applications, vol. 299, no. 2, pp. 663-675, 2004.

[31] Z. Teng, Y. Zhang, and S. Gao, "Permanence criteria for general delayed discrete nonautonomous $n$-species Kolmogorov systems and its applications," Computers \& Mathematics with Applications, vol. 59, no. 2, pp. 812-828, 2010.

[32] J. Dhar and K. S. Jatav, "Mathematical analysis of a delayed stage-structured predator-prey model with impulsive diffusion betweentwo predators territories," Ecological Complexity. In press.

[33] S. Liu and L. Chen, "Necessary-sufficient conditions for permanence and extinction in Lotka-Volterra system with distributed delays," Applied Mathematics Letters, vol. 16, no. 6, pp. 911-917, 2003.

[34] X. Liao, S. Zhou, and Y. Chen, "Permanence and global stability in a discrete $n$-species competition system with feedback controls," Nonlinear Analysis: Real World Applications, vol. 9, no. 4, pp. 1661-1671, 2008.
[35] H. Hu, Z. Teng, and H. Jiang, "On the permanence in nonautonomous Lotka-Volterra competitive system with puredelays and feedback controls," Nonlinear Analysis: Real World Applications, vol. 10, no. 3, pp. 1803-1815, 2009.

[36] Y. Muroya, "Permanence and global stability in a Lotka-Volterra predator-prey system with delays," Applied Mathematics Letters, vol. 16, no. 8, pp. 1245-1250, 2003.

[37] T. Kuniya and Y. Nakata, "Permanence and extinction for a nonautonomous SEIRS epidemic model," Applied Mathematics and Computation, vol. 218, no. 18, pp. 9321-9331, 2012.

[38] Z. Hou, "On permanence of Lotka-Volterra systems with delays and variable intrinsic growth rates," Nonlinear Analysis: Real World Applications, vol. 14, no. 2, pp. 960-975, 2013.

[39] C.-H. Li, C.-C. Tsai, and S.-Y. Yang, "Analysis of the permanence of an SIR epidemic model with logistic process and distributed time delay," Communications in Nonlinear Science and Numerical Simulation, vol. 17, no. 9, pp. 3696-3707, 2012.

[40] F. Chen and M. You, "Permanence for an integrodifferential model of mutualism," Applied Mathematics and Computation, vol. 186, no. 1, pp. 30-34, 2007.

[41] X. Lv, S. Lu, and P. Yan, "Existence and global attractivity of positive periodic solutions of Lotka-Volterra predator-prey systems with deviating arguments," Nonlinear Analysis: Real World Applications, vol. 11, no. 1, pp. 574-583, 2010.

[42] F. Montes de Oca and M. Vivas, "Extinction in two dimensional Lotka-Volterra system with infinite delay," Nonlinear Analysis. Real World Applications, vol. 7, no. 5, pp. 1042-1047, 2006.

[43] T. Yoshizawa, Stability Theory by Liapunov's Second Method, The Mathematical Society of Japan, Tokyo, Japan, 1966. 


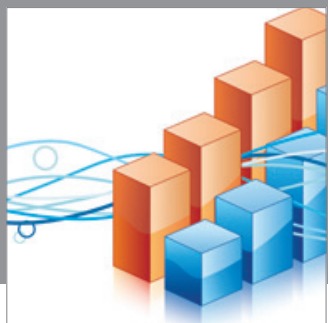

Advances in

Operations Research

mansans

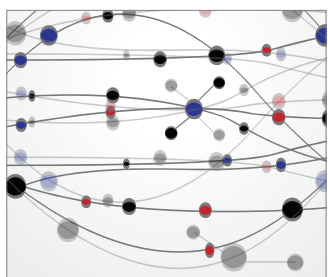

The Scientific World Journal
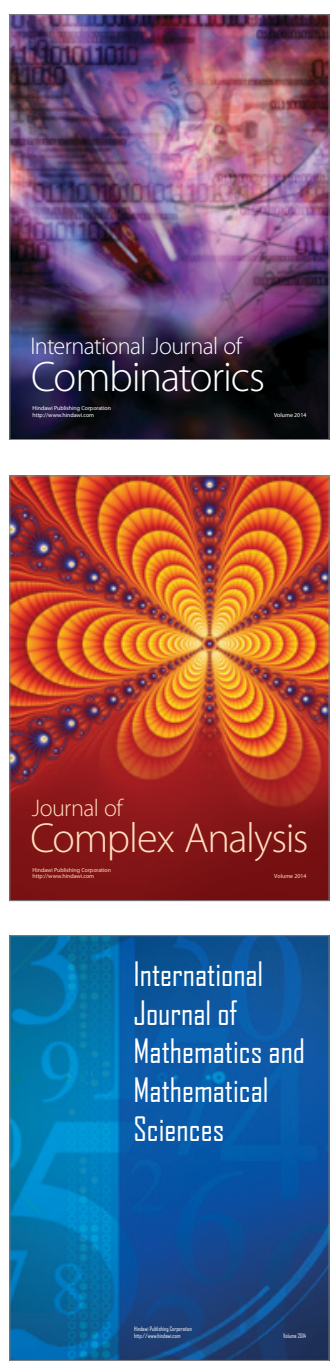
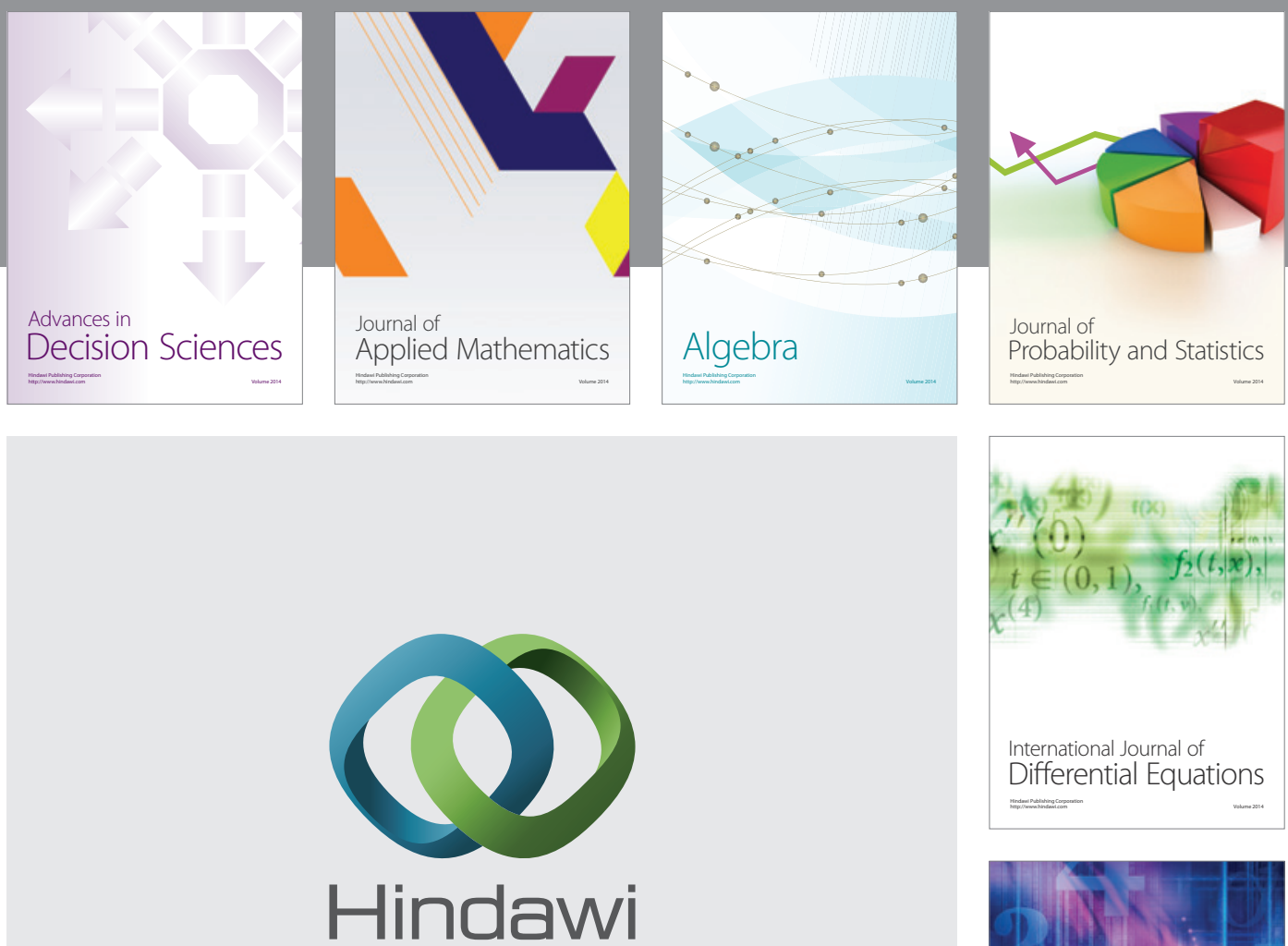

Submit your manuscripts at http://www.hindawi.com
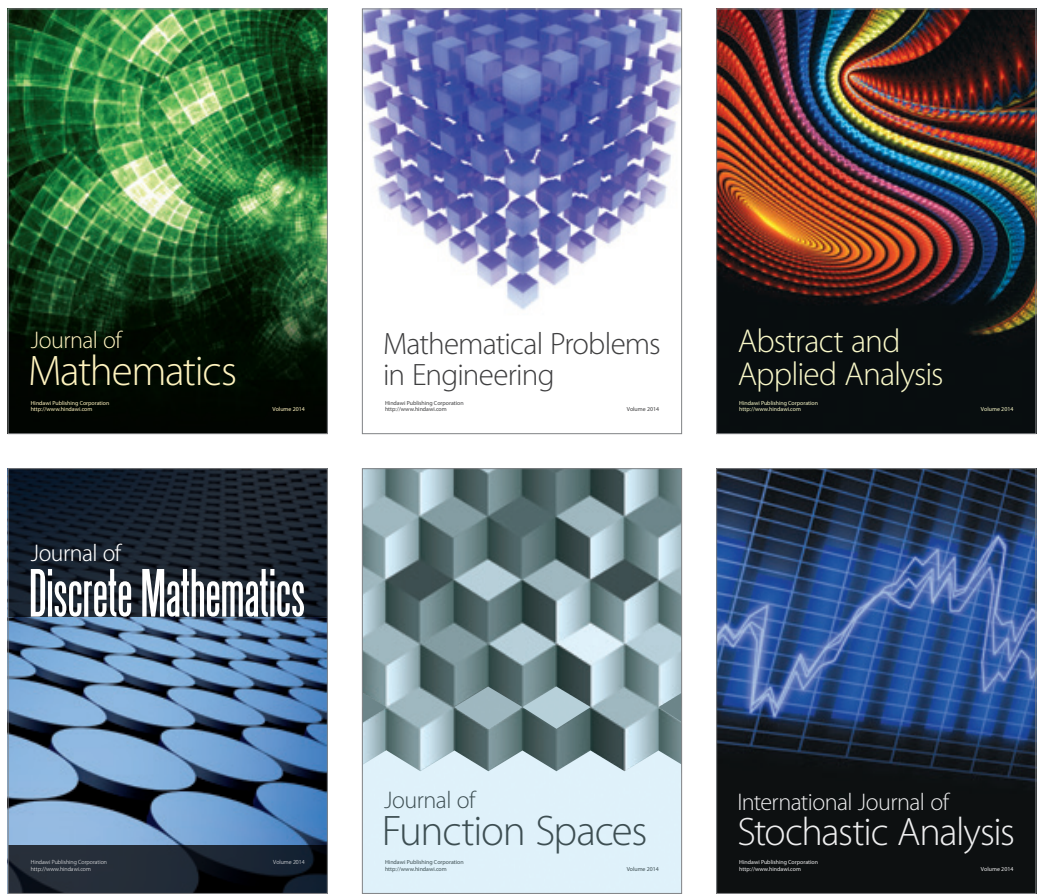

Journal of

Function Spaces

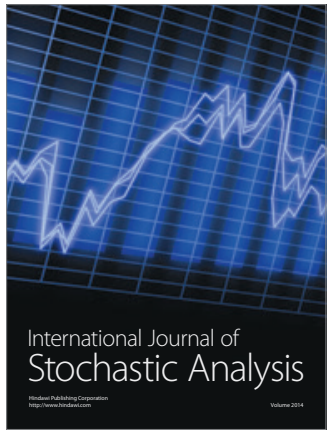

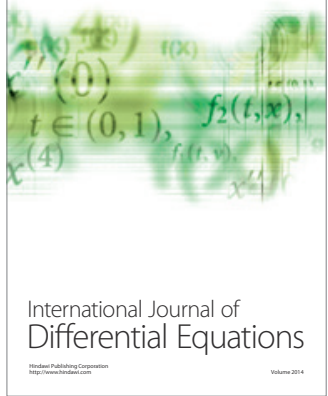
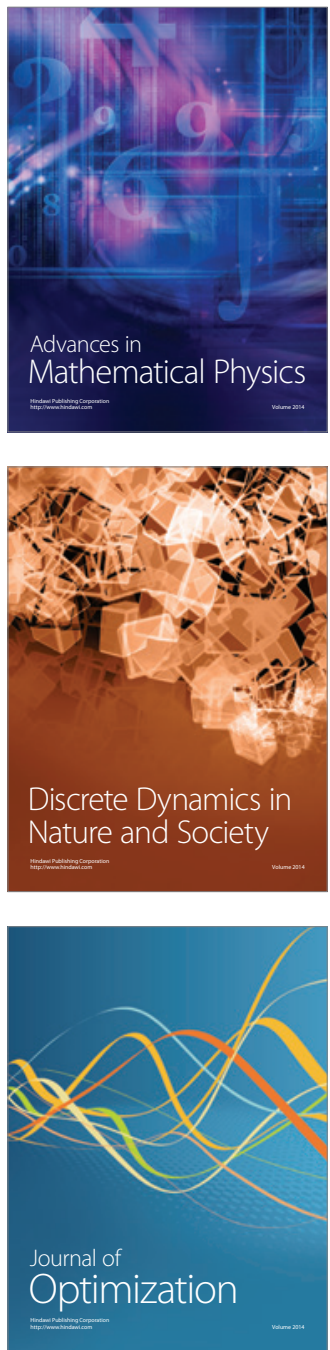